\title{
Genetic Variation in Photosynthetic Responses to Chilling Modulates Proton Motive Force, Cyclic Electron Flow and Photosystem II Photoinhibition
}

\author{
Donghee Hoh ${ }^{1}$, Isaac Osei-Bonsu ${ }^{2}$, Abhijnan Chattopadhyay ${ }^{3}$, Atsuko Kanazawa ${ }^{1}$, Nicholas \\ Fisher $^{1}$, Oliver Tessmer ${ }^{1}$, Jeffrey Cruz ${ }^{3}$, Philip Roberts ${ }^{4}$, Bao Lam Huynh ${ }^{4}$, David Hall ${ }^{1}$, \\ and David M. Kramer ${ }^{3}$ \\ ${ }^{1}$ Michigan State University \\ ${ }^{2}$ Crops Research Institute \\ ${ }^{3}$ MSU-DOE Plant Research Laboratory \\ ${ }^{4}$ University of California Riverside
}

October 14, 2021

\begin{abstract}
The work demonstrates the use of detailed, high-throughput phenotyping to generate and test mechanistic models to explain the genetic diversity of photosynthetic responses to abiotic stress. We assessed a population of recombinant inbred lines (RILs) of cowpea (Vigna unguiculata. (L.) Walp.) with significant differences in a range of photosynthetic responses to chilling. We found well-defined, colocalized (overlapping) QTL intervals for photosynthetic parameters, suggesting linkages among the redox states of $\mathrm{Q}_{\mathrm{A}}$, the thylakoid pmf, through effects on cyclic electron flow and photodamage to PSII. We propose that these genetic variations optimize photosynthesis in the tolerant lines under low temperatures, preventing recombination reactions within Photosystem II that can lead to deleterious ${ }^{1} \mathrm{O}_{2}$ production. By contrast, we did not observe linkages to PSI redox state, PSI photodamage or ATP synthase activity, or nyctinastic (diurnally controlled) leaf movements, likely indicating that several proposed models likely do not contribute to the genetic control of photosynthesis at low temperature in our mapping panel. The identified QTL intervals include a range of potential causative genetic components, with direct applications to breeding of photosynthesis for more climate-resilient productivity.
\end{abstract}

\section{Hosted file}

Genetic Variation in Photosynthetic Responses to Chilling - submitted.pdf available at https://authorea.com/users/441110/articles/541693-genetic-variation-in-photosyntheticresponses-to-chilling-modulates-proton-motive-force-cyclic-electron-flow-andphotosystem-ii-photoinhibition 